\section{B-Cell Lymphoma Involving the Biliary Tree}

Primary or secondary non-Hodgkin's lymphomas of the extrahepatic bile ducts are rare findings. The diagnosis can be established by means of exfoliative bile cytology, brush cytology, and biopsy, including immunohistochemistry and immunocytochemistry via endoscopic retrograde cholangiography, or percutaneous transhepatic cholangiography.

In an 84-year-old patient with jaundice, known to have a stage-III B-cell chronic lymphatic leukemia (B-CLL), sonography and CT confirmed lymph node enlargement in the porta hepatis, hepatosplenomegaly, and dilated intrahepatic bile ducts. Endoscopic a, b

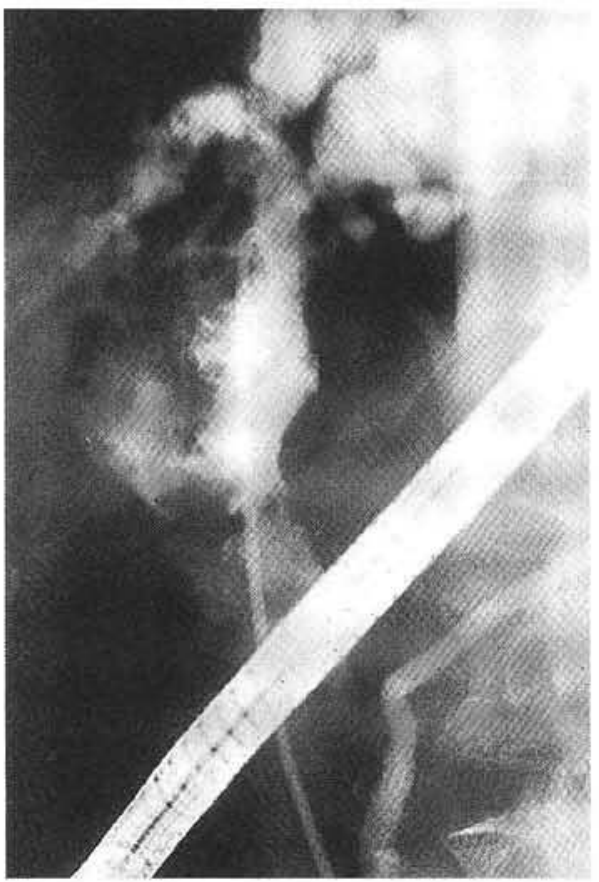

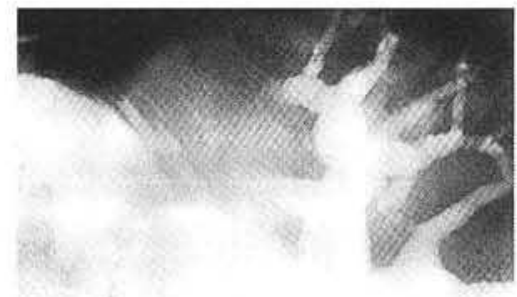

Figure 1a: Common bile duct with narrowed and dilated segments. $\mathbf{b}$ Dilated intrahepatic bile ducts, visible after insertion of a nasobiliary tube. 
retrograde cholangiopancreatography (ERCP) revealed massive dilation of the intrahepatic bile ducts and the biliary duct showed narrowed and dilated segments (Figure 1). After endoscopic papillotomy (EPT), cytological and biopsy tests were performed. Brush cytology and exfoliative bile cytology resembled a peripheral blood smear in B-CLL. The histology and immunohistology confirmed a B-CLL involving the common bile duct. The small lymphocytes and lymphoplasmacellular elements expressed CD$19,20,22,24$ and CD-5. There was no evidence of cytomegalovirus or mycobacterial infection. Serological tests for cytomegalovirus, anti-neutrophile cytoplasmatic antibodies (ANCA), and human immunodeficiency virus as well as the tuberculin skin reaction, were negative.

The sensitivity of brush cytology via ERCP in the diagnosis of biliary and pancreatic carcinoma is $56.2 \%$; the specificity is $100 \%$ (1). In the diagnosis of malignant bile duct strictures, the sensitivity of brush cytology was $59 \%$ and that of exfoliative bile cytology was $24 \%$ (2).

Non-Hodgkin's lymphoma of the bile ducts is rare. Broulard et al. (3) described a primary high-grade malignant T-cell-rich B-CLL of the common bile duct in a 34-year-old woman; the diagnosis was made by laparotomy. Wegerle et al (4) reported a 28 -year-old man with angioimmunoblastic lymphadenopathy or T-zone lymphoma of the bile ducts, initially diagnosed as primary sclerosing cholangitis. Moody reported three patients developing NonHodgkin's lymphoma in the porta hepatis after orthotopic liver transplantation (5).

Jaundice in malignant lymphoma can be caused by liver involvement, bile duct obstruction by lymph nodes or malignant bile duct involvement. Another rare cause of jaundice is the "vanishing bile duct syndrome" in Hodgkin's disease, where the diagnosis is confirmed by liver biopsy. Viral, bacterial, and parasitic infections, as well as secondary malignancy, should be considered in an immunocompromised host. Secondary autoimmune diseases of the liver, and toxic effects of chemotherapy and radiotherapy in NonHodgin's lymphoma can cause intrahepatic or extrahepatic jaundice.

The diagnosis of bile duct involvement by a Non-Hodgkin's lymphoma can be established by combining brush cytology, exfoliative bile cytology, and biopsy from the bile ducts via endoscopic retrograde cholangiography or percutaneous transhepatic cholangiography. In leukemic non-Hodgkin's lymphoma, biopsy may be the best diagnostic approach, as cytology specimens can be contaminated with blood. Most patients present with secondary bile duct involvement in advanced-stage non-Hodgkin's lymphoma. Although bile duct locations are very rare, they should be included in the differential diagnosis of obstructive jaundice in non-Hodgkin's lymphoma patients.

\section{Meuthen, W. Hummerich, M. Krakau, C. Mauel \\ Medical Clinic, Holweide Hospital, Cologne City Hospitals, \\ Cologne, Germany}

\section{References}

1. Ferrari AP Jr, Lichtenstein DR, Slivka A, Chang C, CarrLocke DR. Brush cytology during ERCP for the diagnosis of biliary and pancreatic malignancies. Gastrointest Endosc 1994; 40: $140-5$.

2. Kurzawinski T, Deery A, Dooley J, Dick R, Hobbs K, Davidson B. A prospective controlled study comparing brush cytology and bile exfoliative cytology for diagnosing bile duct structures. Gut 1992; 33: 1675-7.

3. Broulard JP, Molinard J, Nemeth J, Valleur P, Galian A. Primary T-cell lymphoma of the common bile duct. Virchows Arch A Pathol Anat Histopathol 1993; 423: 513-7.

4. Wegerle W, Garbrecht M, Nerl C, Schmitt W. Angioimmunoblastic lymphadenopathy with dysproteinemia and sclerosing cholangitis. Dtsch Med Wochenschr 1994; 119: 332-7.

5. Moody AR, Wreson SR, Greig PD. Non-Hodgkin lymphoma in the porta hepatis after orthotopic liver transplantation. Radiology 1992; 182: 867-70.

Corresponding Author

I. Meuthen, M.D.

Medizinische Klinik

Krankenhaus Holweide

Kliniken der Stadt Köln

Neufelderstrasse 32

$51067 \mathrm{Köln}$

Germany

Fax: +49-221-6781 2799 